Supporting Information

\title{
Probing Charged Aqueous Interfaces Near Critical Angles: Effect of Varying Coherence Length
}

\author{
Dennis K. Hore ${ }^{1}$ and Eric Tyrode ${ }^{2}$ \\ ${ }^{1}$ Department of Chemistry, University of Victoria, Victoria, Canada, V8W 3 V6 \\ ${ }^{2}$ Department of Chemistry, KTH Royal Institute of Technology, SE-10044 Stockholm, Sweden \\ Corresponding authors: dkhore@uvic.ca and tyrode@kth.se
}

\section{Content:}

Model of distance-dependent potential

Pages S2-S3

Comparison of $f_{3}$ and $g_{3}$ profiles

Pages S4-S5

Justification of parameters used in the fused silica acid-base model

Page S6

Local field correction of experimental data

Pages S6-S8

Normalization of experimental data

Page S9

Evaluating the relative phase between $\chi^{(2)}$ and $\chi^{(3)}$

Pages S10-S12 


\section{Model of distant dependent potential}

Fig. S1 provides a comparison between the linearized and full-form of the distance-dependent potential evaluated at $10^{-2} \mathrm{M}, 10^{-4} \mathrm{M}$ and $10^{-6} \mathrm{M}$, plotted for the first $1 \mu \mathrm{m}$ into the solution from the silica surface. One notices that the agreement is good at low ionic strength, but at higher ionic strength the actual potential (solid lines) decays more rapidly than predicted by the linearized model (dashed lines).

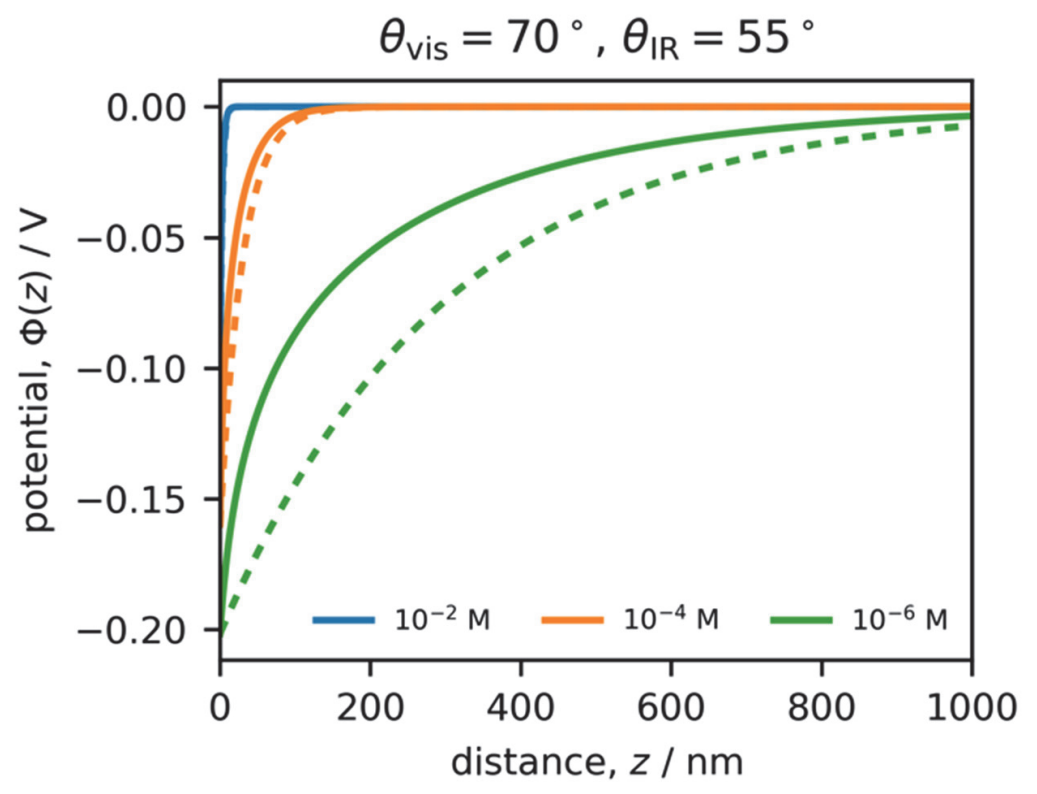

Fig. S1. Comparison between the linearized (dashed lines) and full-form (solid lines) of the distancedependent potential $\Phi(z)$ for a few different ionic strengths. 
There are two options for the integration along z, a numerical approach with a small step size in evaluating the Riemann sum, or the series expansion evaluated to a sufficiently large number of terms. Fig. S2 illustrates the comparison of these approaches. While the first $\sim 10$ terms are sufficient at high ionic strengths, the low ionic strength agreement is achieved only with $\sim 100$ terms.
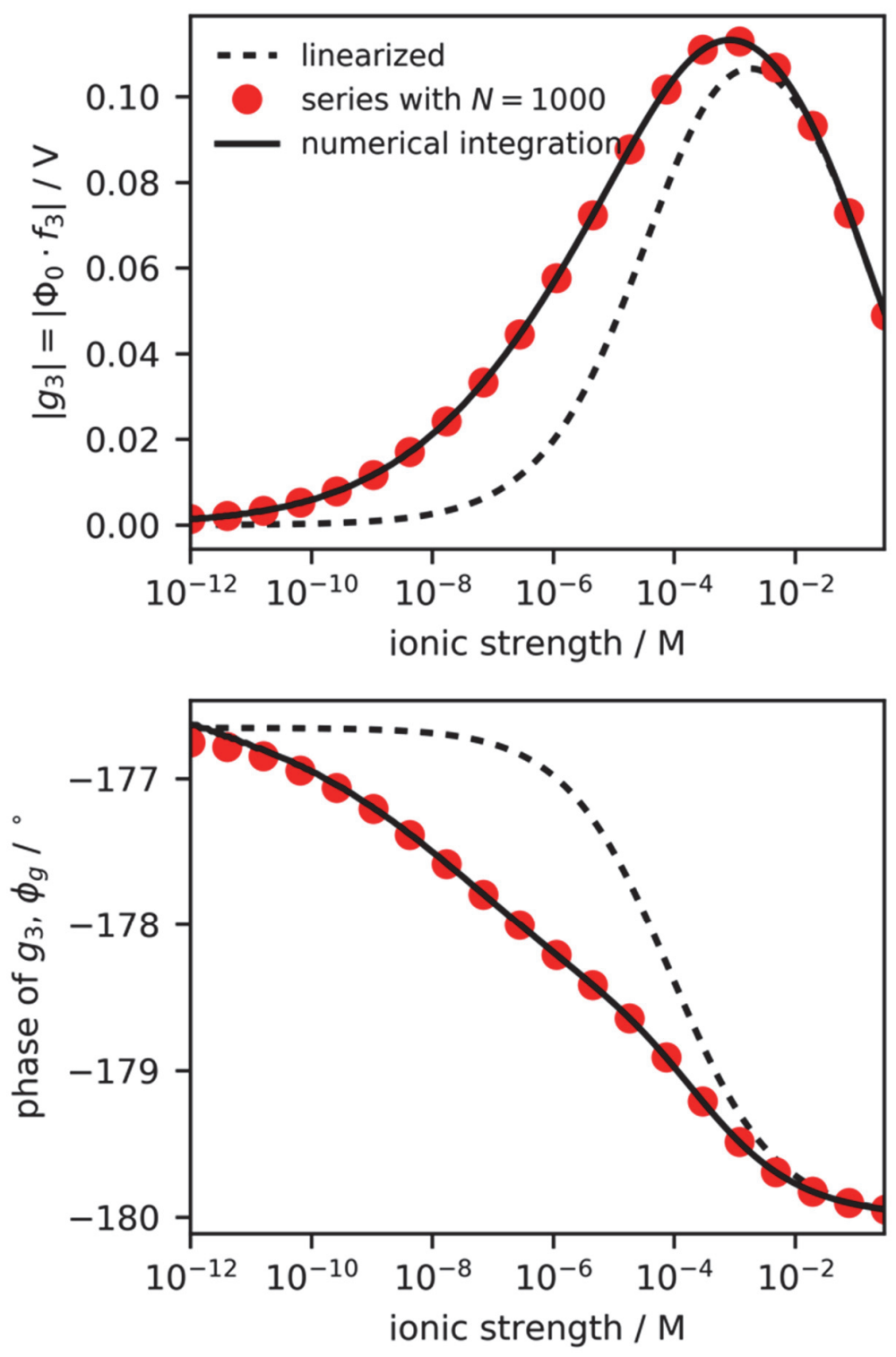

Fig. S2. Comparison between linearized (dashed lines) and full-form obtained through numerical integration (solid line) and the series solution evaluated to the first 1000 terms (red circles). 


\section{Comparison of $f_{3}$ and $g_{3}$ profiles}

In the linearized Guoy-Chapman model, $P^{(3)}=f_{3} \Phi_{0} \chi^{(3)}$ and upon using the complete expression for $\Phi(z)$ we have obtained $P^{(3)}=g_{3} \chi^{(3)}$. As a result, $g_{3}$ and $f_{3}$ cannot be compared directly, but a comparison can be made between $g_{3}$ and $\Phi_{0} f_{3}$. For completeness, and to understand the difference in properties from $f_{3}$ that has been more extensively described in the literature, we include all 3 cases, with $g_{3}$ in the manuscript Fig. $4, f_{3}$ in Fig. S3, and $f_{3} \Phi_{0}$ in Fig. S4.
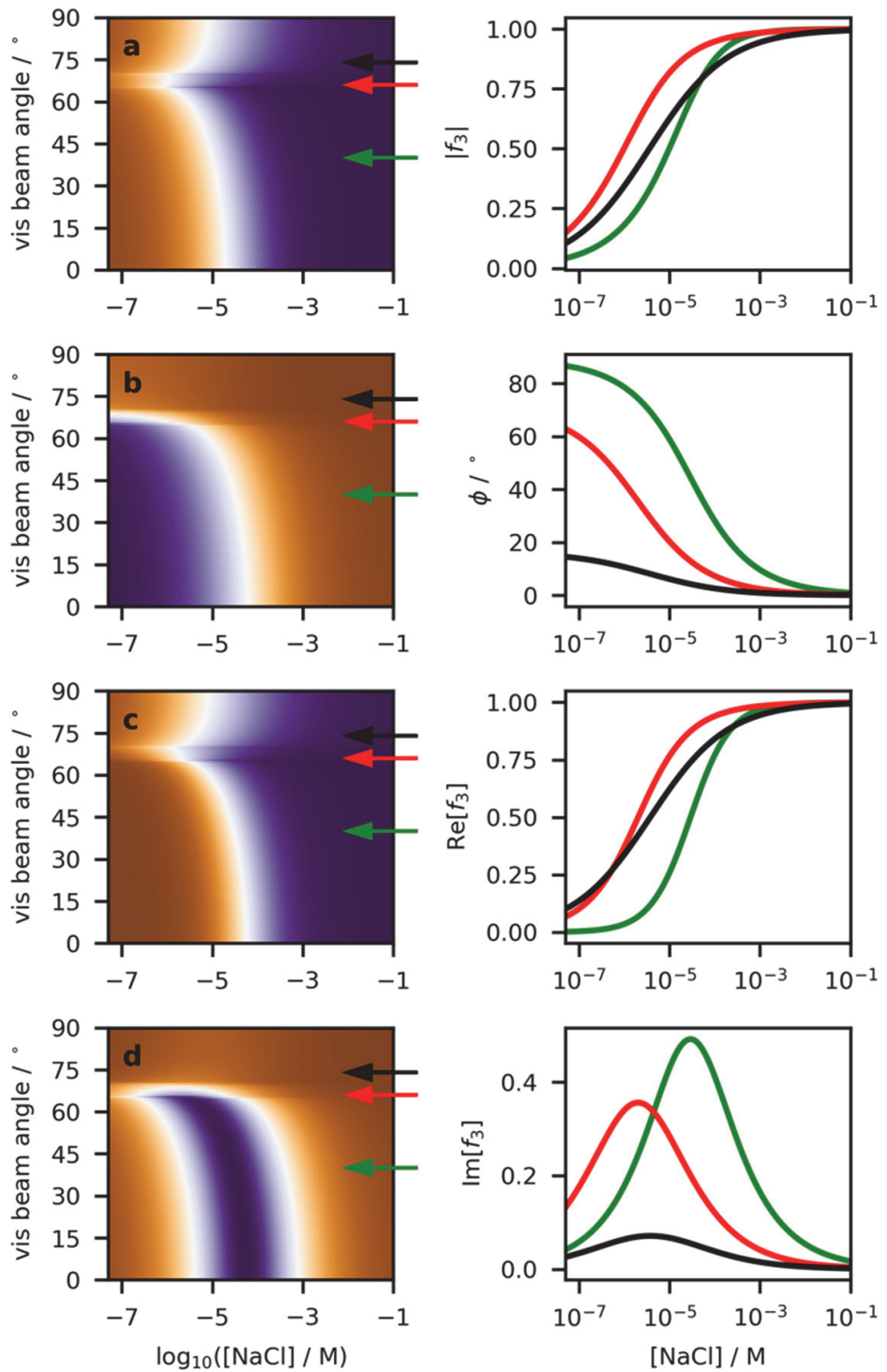

Fig. S3. Values of the (a) magnitude, (b) phase, (c) real and (d) imaginary components of $f_{3}$ as a function of the ionic strength and angle of incidence of the visible beam. In all cases the incoming IR beam was fixed at $50^{\circ}$. A slice through the $40^{\circ}$ incident visible angle is shown in green, $66^{\circ}$ in red, and $74^{\circ}$ in black. Numerical values corresponding to the minimum (darkest orange) and maximum (darkest purple) colors are the same as the extent of the axes in the corresponding plots with the slices in the right column. 

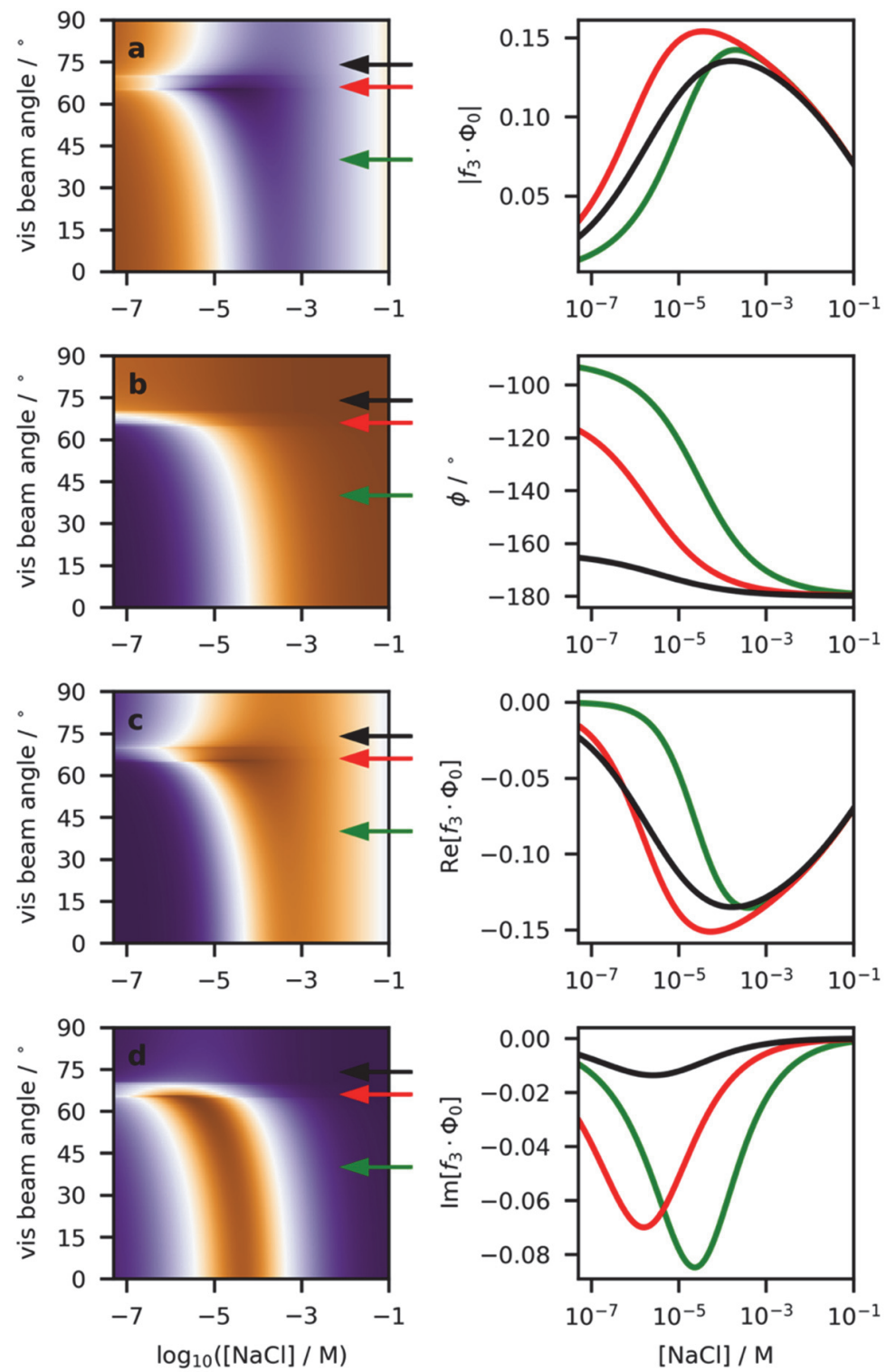

Fig. S4. Values of the (a) magnitude, (b) phase, (c) real and (d) imaginary components of $f_{3} \Phi_{0}$ as a function of the ionic strength and angle of incidence of the visible beam. In all cases the incoming IR beam was fixed at $50^{\circ}$. A slice through the $40^{\circ}$ incident visible angle is shown in green, $66^{\circ}$ in red, and $74^{\circ}$ in black. Numerical values corresponding to the minimum (darkest orange) and maximum (darkest purple) colors are the same as the extent of the axes in the corresponding plots with the slices in the right column. 


\section{Justification of parameters used in the fused silica acid-base model}

For calculating the surface potential dependence with ionic strength using the Gouy Chapman model, a pK $\mathrm{pK}_{\mathrm{a}}$ of 4 and area per silanol group of $200 \AA^{2}$ were assumed. These values are justified as follows: The typical silanol density for amorphous or fused silica is 4.6-4.9 silanols $/ \mathrm{nm}^{2},{ }^{1}$ which corresponds to $\sim 21 \AA^{2} /$ silanol. However, only $\sim 25 \%$ actually deprotonate at $\mathrm{pH} 10$. $^{2}$ Thus relevant area is $\sim 90 \AA^{2} /$ silanol. Moreover, silica have been shown to have at least two different $\mathrm{p} K_{\mathrm{a}}$ (i.e. not all types of silanols have the same acidity). ${ }^{3-7}$ Typically, one is suggested to be between $\sim 3.5$ $4.6^{5-7}$ and the other $\sim 8.5-9 .^{5-6}$ In this work, measurements are carried at $\sim \mathrm{pH} 6$ (or $\sim 7$ for pure water), so we consider only the first one to be of relevance, and use an average of the reported values: $\mathrm{p} K_{\mathrm{a}} \sim 4$. Finally, the proportion between the two types of silanols (i.e. $\mathrm{p} K_{\mathrm{a}} \sim 4$ and $\mathrm{p} K_{\mathrm{a}} \sim 9$ ), required for determining the area per silanol in the model, has been reported to lie between 1:2 and $1: 1 .^{5-6}$ When taking the average this results in an $A_{M} \sim 200 \AA^{2} /$ silanol. We note that the calculated surface charge is consistent with those measured at selected ionic strengths for colloidal silica particles. $^{2,8}$

\section{Local field correction of experimental data}

The procedure for correcting the measured SF spectra for local fields, and in particular for the silica/water interface, has been extensively discussed in the literature. ${ }^{9-12}$ Here we used a similar approach, and refer the reader specifically to Ref. ${ }^{11}$ for a detailed description of the equations required. In the calculation, the reported refractive indexes values for water ${ }^{13}$ and silica ${ }^{14}$ were used. The resulting IR frequency dependent local field corrections for the five different experimental geometries used are shown in Fig. S5. Additionally, the gold normalized SF spectra and corresponding local field corrected spectra for the three experimental geometries not included in the main text are shown in Fig. S6, S7, and S8.

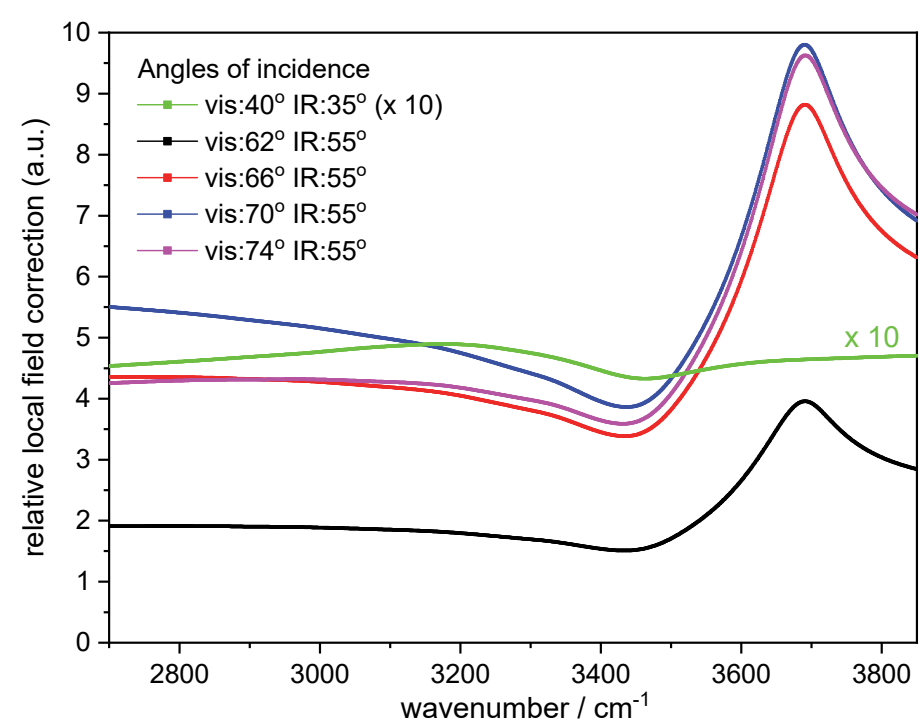

Fig. S5. IR dependence of the local field corrections for each of the experimental geoemetries studied. Note that the values for $40^{\circ}$ visible AOI and $35^{\circ}$ IR AOI experimental geometry, were multiplied by 10 for ease of comparison. The maximum enhancement in the overall spectra intensity is attained close to the critical angles. 

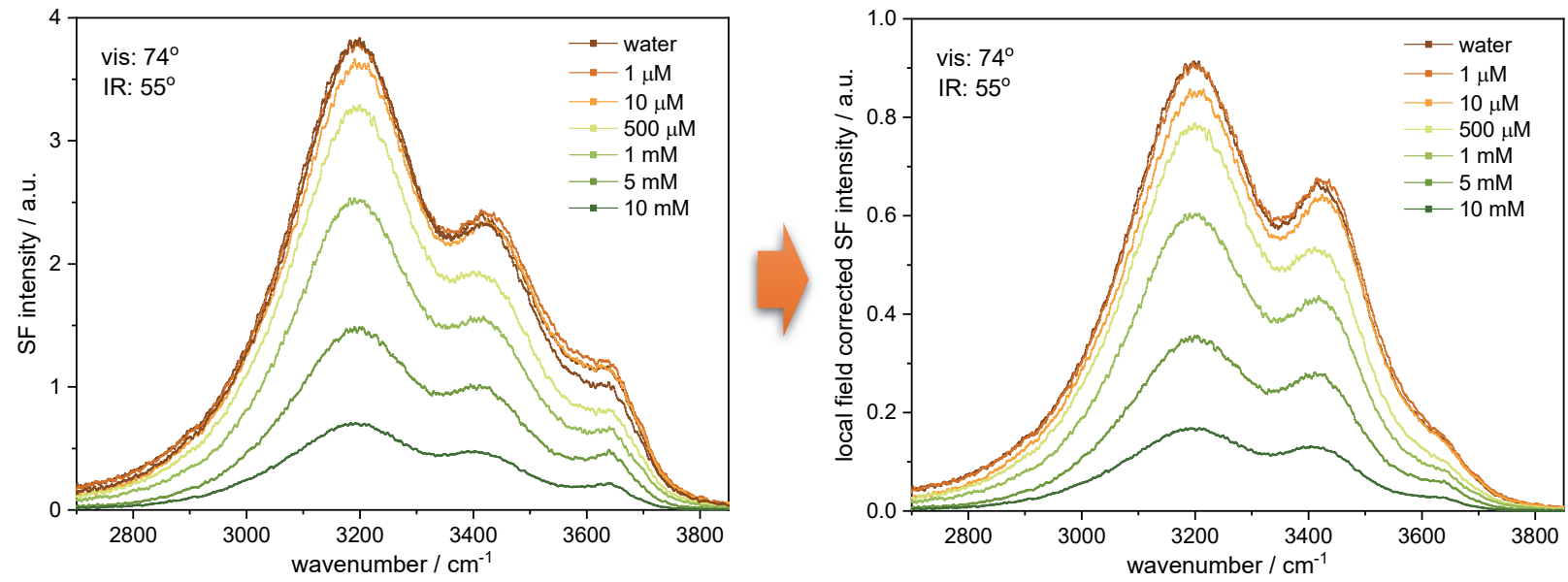

Fig. S6. (left) SF spectra collected in the ssp polarization combination as a function of $\mathrm{NaCl}$ for an IR angle of incidence of $55^{\circ}$ and visible angle of $74^{\circ}$. (right) Local field corrected spectra at the same experimental geometry.
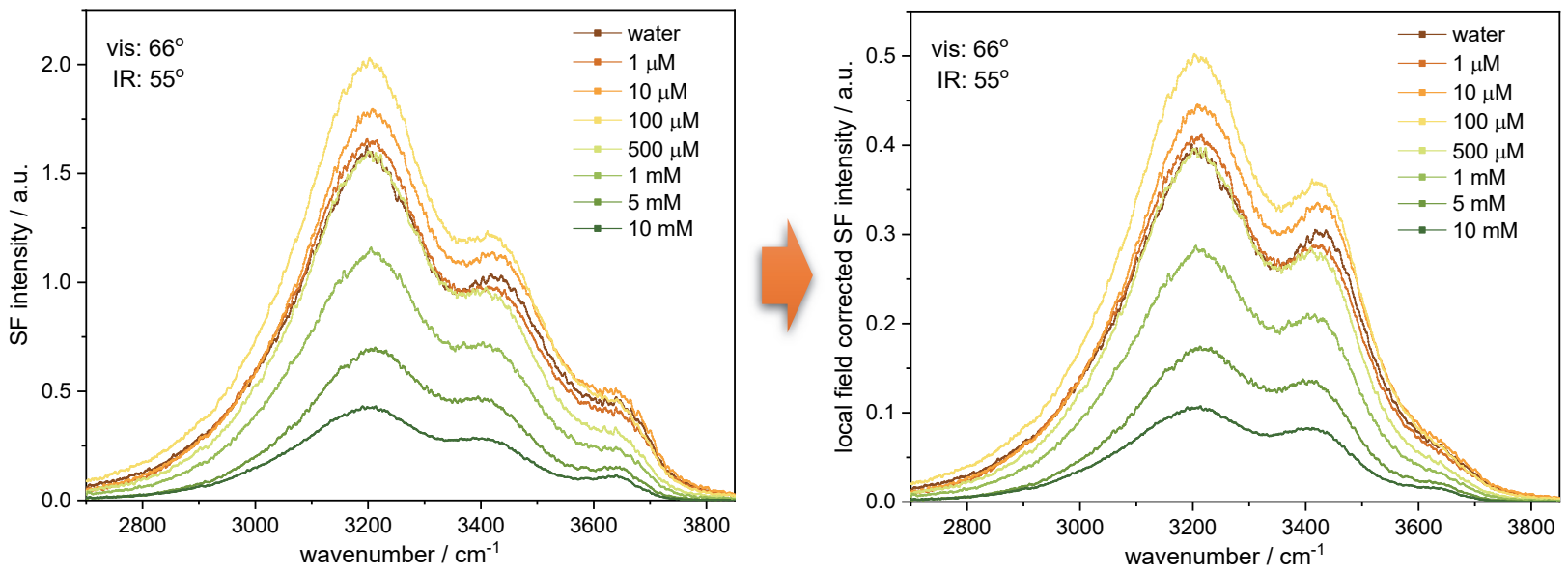

Fig. S7. (left) SF spectra collected in the ssp polarization combination as a function of $\mathrm{NaCl}$ for an IR angle of incidence of $55^{\circ}$ and visible angle of $66^{\circ}$. (right) Local field corrected spectra at the same experimental geometry. 

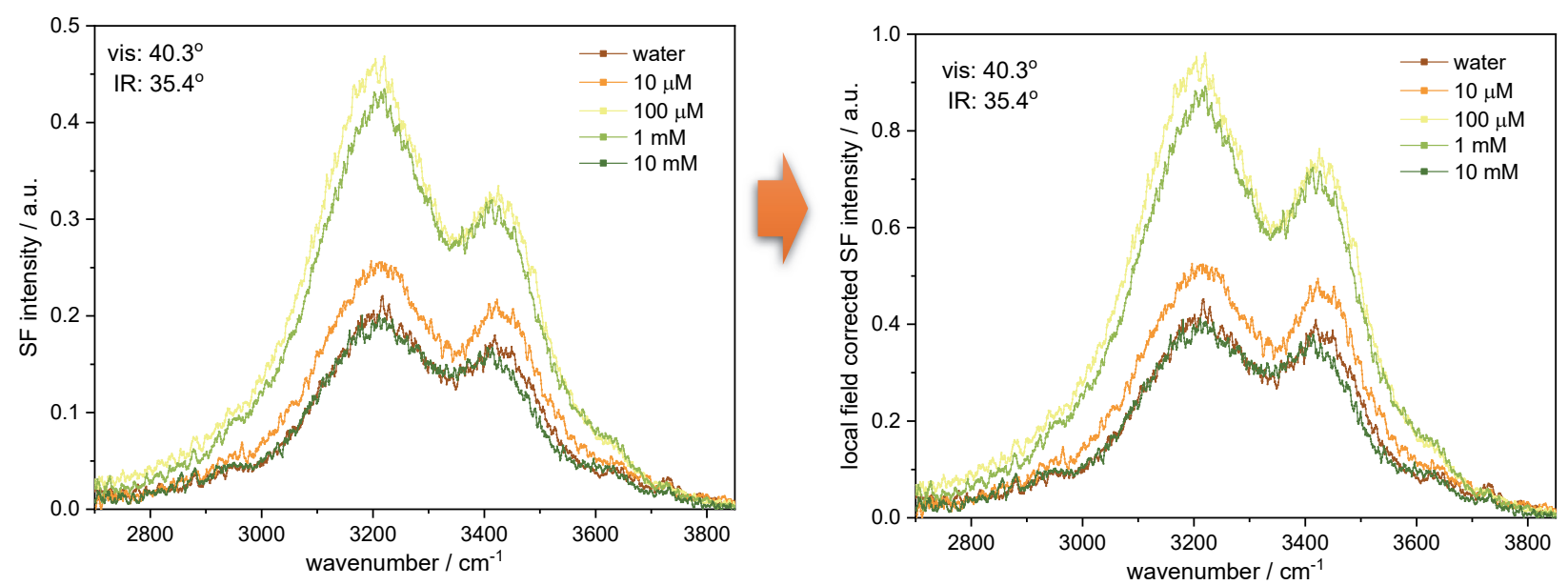

Fig. S8. (left) SF spectra collected in the ssp polarization combination as a function of $\mathrm{NaCl}$ for an IR angle of incidence of $40.3^{\circ}$ and visible angle of $35.4^{\circ}$. (right) Local field corrected spectra at the same experimental geometry. 


\section{Normalization of experimental data}

The relative intensity of data collected at different ionic strengths and beam angles is of primary importance to this study. The experimental data from different experimental geometries was normalized using the following scheme. The procedure is based on the observation that, regardless of which model is used, they all converge at high ionic strengths. Plotted on a logarithmic concentration scale, all models also predict a linear decrease in signal with increasing ionic strength in the region roughly extending between $0.1 \mathrm{mM}$ and $100 \mathrm{mM}$ region. Since a non-linear behavior had been observed at the lower and upper ends of this region, ${ }^{15}$ we have limited in our normalization procedure this range to extend between $1 \mathrm{mM}$ and $5 \mathrm{mM}$. Data points in that region were linearized on a log scale, and the value interpolated at $3 \mathrm{mM}$ was then used to normalize the entire data set.
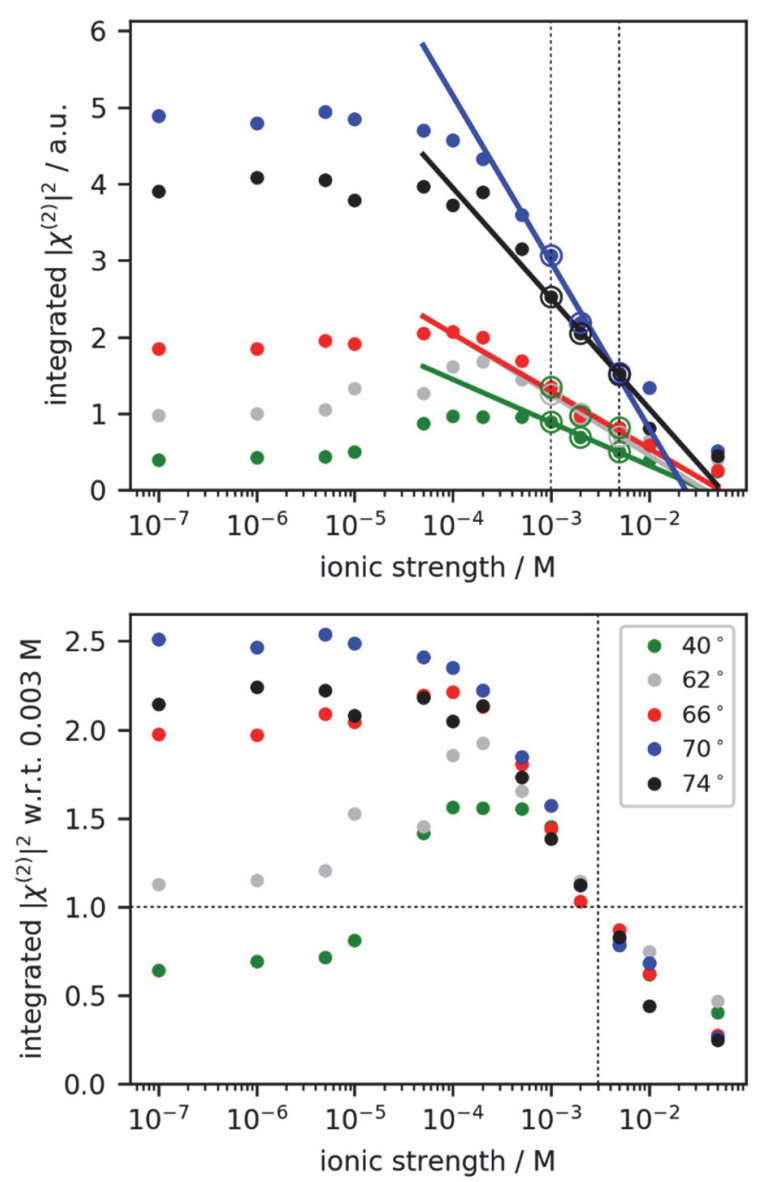

Fig. S9. (top) The raw experimental data, illustrating the region used for normalization (vertical dashed lines), data points considered in the normalization (circled) and the resulting fits (straight lines). (bottom) The scaled data after normalization. 


\section{Evaluating the relative phase between $\chi^{(2)}$ and $\chi^{(3)}$}

In our reduced model, and when we assume a known ionic strength-dependence of the surface potential, there are two unknown parameters, the ratio $R$ between the magnitudes of $\chi^{(2)}$ and $\chi^{(3)}$, and their relative phase $\Delta \phi$. We initially attempted to fit the experimental data to obtain both $R$ and $\Delta \phi$, but found the fitting to be insensitive to $\Delta \phi$ values as long as they were about $\pm 20^{\circ}$ from $\Delta \phi=180^{\circ}$. We have therefore chosen to set $\Delta \phi=180^{\circ}$ and solve for $R$ only. A few illustrative cases are shown below.
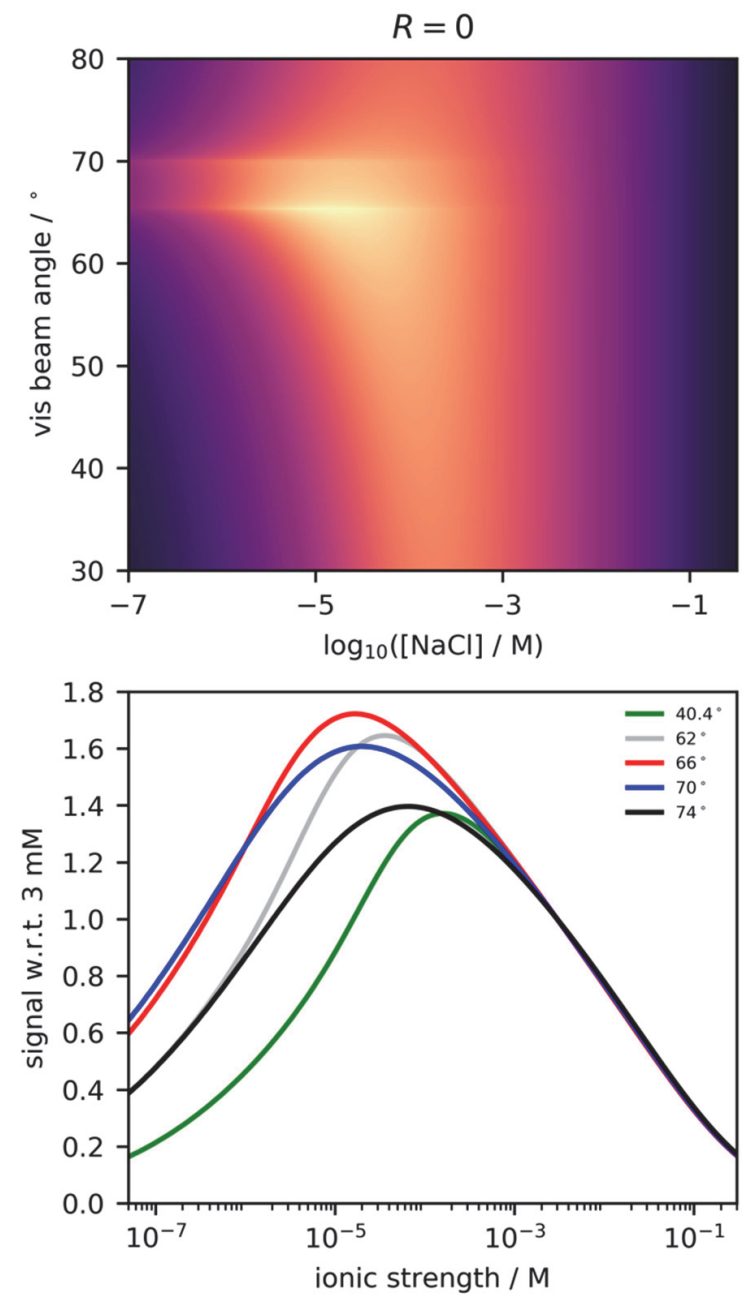

Fig. S10. The effect of setting $R=0$, i.e. the signal is dominated by the $\chi^{(3)}$ contribution. Note that the signal drops at low ionic strength for all angles of incidence. None of the experimental plateaus observed at low ionic strength can be reproduced with this model. 

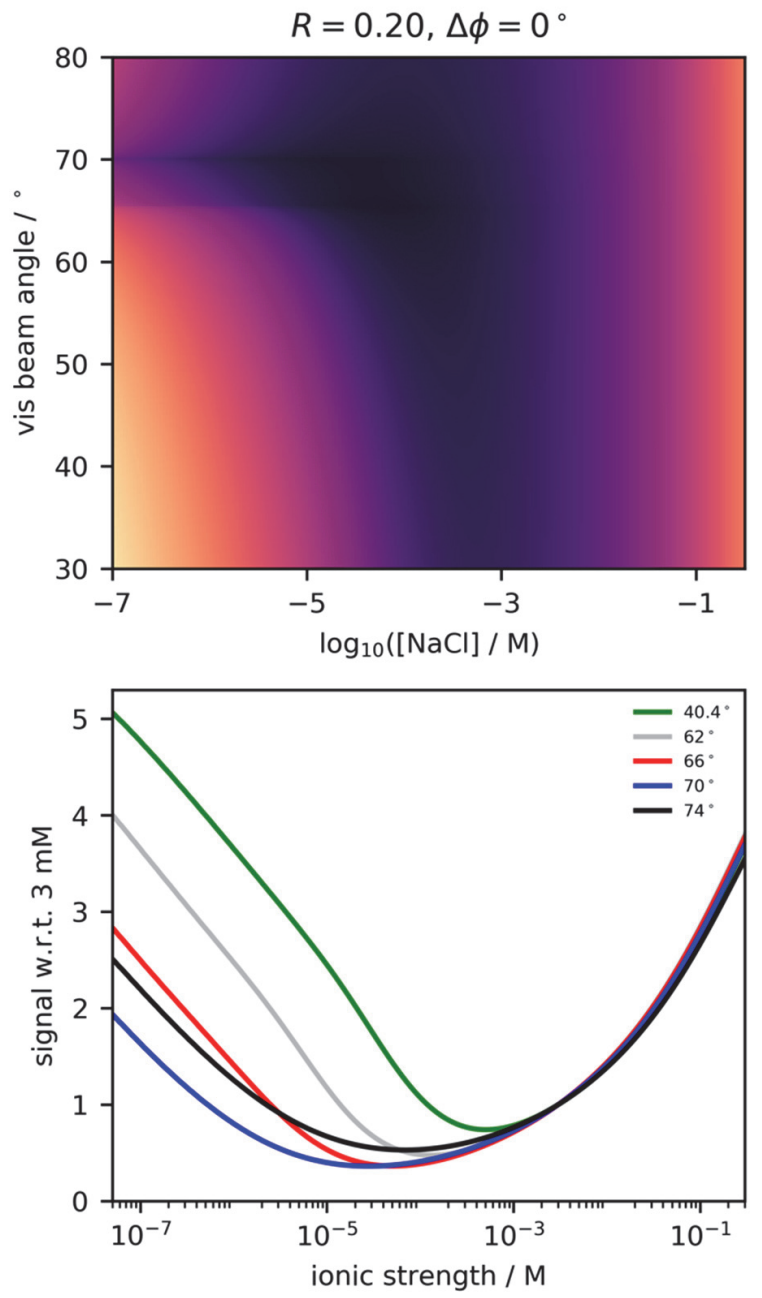

Fig. S11. The predicted intensity as a function of angle and ionic strength with $R=0.2$, and with $\chi^{(2)}$ and $\chi^{(3)}$ in phase. 

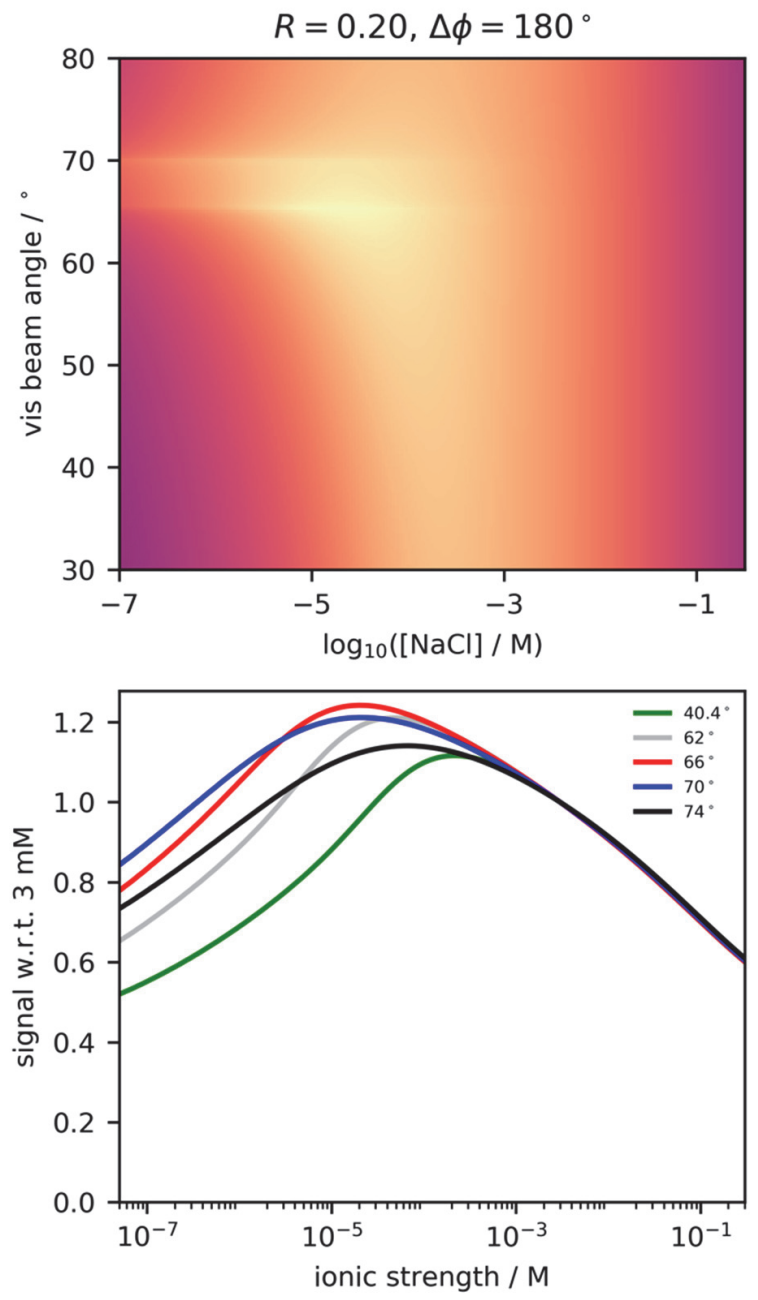

Fig. S12. The predicted intensity as a function of angle and ionic strength with $R=0.2$, but $\chi^{(2)}$ and $\chi^{(3)}$ out of phase with each other. 


\section{References}

1. Zhuravlev, L. T., The Surface Chemistry of Amorphous Silica. Zhuravlev Model. Colloids and Surfaces a-Physicochemical and Engineering Aspects 2000, 173, 1-38.

2. Brown, M. A.; Goel, A.; Abbas, Z., Effect of Electrolyte Concentration on the Stern Layer Thickness at a Charged Interface. Angew. Chem., Int. Ed. Engl. 2016, 55, 3790-3794.

3. Leung, K.; Nielsen, I. M. B.; Criscenti, L., Elucidating the Bimodal Acid-Base Behavior of the Water-Silica Interface from First Principles. Geochim. Cosmochim. Acta 2010, 74, A584A584.

4. Dalstein, L.; Potapova, E.; Tyrode, E., The Elusive Silica/Water Interface: Isolated Silanols under Water as Revealed by Vibrational Sum Frequency Spectroscopy. Phys. Chem. Chem. Phys 2017, 19, 10343-10349.

5. Ong, S.; Zhao, X.; Eisenthal, K. B., Polarization of Water Molecules at a Charged Interface: Second-Harmonic Studies of the Silica/Water Interface. Chem. Phys. Lett. 1992, 191, $327-$ 35 .

6. Darlington, A. M.; Gibbs-Davis, J. M., Bimodal or Trimodal? The Influence of Starting Ph on Site Identity and Distribution at the Low Salt Aqueous/Silica Interface. J. Phys. Chem. C 2015, 119, 16560-16567.

7. Méndez, A.; Bosch, E.; Rosés, M.; Neue, U. D., Comparison of the Acidity of Residual Silanol Groups in Several Liquid Chromatography Columns. Journal of Chromatography A 2003, 986, 33-44.

8. Brown, M. A.; Abbas, Z.; Kleibert, A.; Green, R. G.; Goel, A.; May, S.; Squires, T. M., Determination of Surface Potential and Electrical Double-Layer Structure at the Aqueous Electrolyte-Nanoparticle Interface. Phys. Rev. X 2016, 6, 011007.

9. T.F.Heinz, Second-Order Nonlinear Optical Effects at Surfaces and Interfaces. In Nonlinear Surface Electromagnetic Phenomena, H.E.Ponath; G.I.Stegeman, Eds. Amsterdam, 1991; pp 353-416.

10. Shen, Y. R., Optical Second Harmonic Generation at Interfaces. Annu. Rev. Phys. Chem. 1989, 40, 327-350.

11. Liljeblad, J. F. D.; Tyrode, E., Vibrational Sum Frequency Spectroscopy Studies at Solid/Liquid Interfaces: Influence of the Experimental Geometry in the Spectral Shape and Enhancement. J. Phys. Chem. C 2012, 116, 22893-22903.

12. Tyrode, E.; Liljeblad, J. F. D., Water Structure Next to Ordered and Disordered Hydrophobic Silane Monolayers: A Vibrational Sum Frequency Spectroscopy Study. J. Phys. Chem. C 2013, 117, 1780-1790.

13. Segelstein, D. J. The Complex Refractive Index of Water. Masters, University of Missouri, Kansas City, 1981.

14. Malitson, I. H., Interspecimen Comparison of the Refractive Index of Fused Silica. J. Opt. Soc. Am. 1965, 55, 1205-1208.

15. Jena, K. C.; Hore, D. K., Variation of Ionic Strength Reveals the Interfacial Water Structure at a Charged Mineral Surface. J. Phys. Chem. C 2009, 113, 15364-15372. 